\title{
One-qubit fingerprinting schemes
}

\author{
J. Niel de Beaudrap* \\ Department of Computer Science, University of Calgary, Calgary, Alberta, Canada T2N 1N4
}

(Dated: August 26, 2018)

\begin{abstract}
Fingerprinting is a technique in communication complexity in which two parties (Alice and Bob) with large data sets send short messages to a third party (a referee), who attempts to compute some function of the larger data sets. For the equality function, the referee attempts to determine whether Alice's data and Bob's data are the same. In this paper, we consider the extreme scenario of performing fingerprinting where Alice and Bob both send either one bit (classically) or one qubit (in the quantum regime) messages to the referee for the equality problem. Restrictive bounds are demonstrated for the error probability of one-bit fingerprinting schemes, and show that it is easy to construct one-qubit fingerprinting schemes which can outperform any one-bit fingerprinting scheme. The author hopes that this analysis will provide results useful for performing physical experiments, which may help to advance implementations for more general quantum communication protocols.
\end{abstract}

PACS numbers: 03.67.Hk, 03.67.-a

\section{INTRODUCTION}

Fingerprinting is a useful mechanism for computing functions of large and distributed sets of data, by sending short messages called 'fingerprints' of the data to a third party for computation. The aim is to reduce the amount of communication, at the cost of some manageable probability of error.

The notion of fingerprinting arises naturally in the setting of communication complexity (see [1]). In the simultaneous message passing model, which was introduced by Yao in his original paper on communication complexity 2], two parties (Alice and Bob) each have a piece of data represented by a string ( $\alpha$ for Alice and $\beta$ for Bob). They wish to compute some function $f$ of their strings, $f(\alpha, \beta)$. However, we constrain Alice and Bob in the following way: they may not communicate with one another, but they may each send one message to a third party (a referee) who will attempt to compute $f(\alpha, \beta)$ using messages sent by Alice and Bob. It is assumed that Alice, Bob, and the referee have established some protocol which describes:

- how Alice and Bob choose messages to send to the referee depending on their strings, and

- how the referee interprets the messages in order to attempt to compute $f(\alpha, \beta)$.

In our scenario, we are assuming Alice and Bob do not have access to a common source of random bits. A simple protocol in which Alice and Bob simply transmit (a binary representation of) their entire strings will suffice. The question that is asked is: how great a probability of success can they attain if they each transmit less information to the referee? We refer to the shorter messages that Alice and Bob send as fingerprints of their data.

${ }^{*}$ Email: jd@cpsc.ucalgary.ca
In the case where Alice and Bob wish to compare their strings for equality, we may use $f(\alpha, \beta)=\delta_{\alpha \beta}$, which evaluates to 0 if $\alpha=\beta$ and 1 otherwise.

Performing fingerprinting using quantum information was first investigated for the asymptotic case in 3]. In that paper, it was shown that fingerprints of length $O(\log n)$ suffice for determining whether or not two $n$ bit strings are equal, with an error probability less than $\varepsilon$ (for any constant $\varepsilon>0$ ). This contrasts with the classical case, where it is known that fingerprints of size $\Omega(\sqrt{n})$ are necessary for comparable performance [4]. The result of [3] is part of a sequence of results including [5, 6], where it has been shown that communication complexity can be reduced using quantum communication. Although these results demonstrate asymptotic savings, they do not explicitly demonstrate savings in small instances that might be suitable for experimental implementations in order to verify the theory. For example, it is not clear that the significant quantum/classical communication separations shown in [5] and [6] hold for particularly small problemsize instances.

In this paper, we examine very small instances of fingerprinting. Specifically, if Alice and Bob may only transmit one bit (or one qubit) of information to the referee, what can we say about the probability of success in determining whether $\alpha=\beta$ ? By analysing these very limited instances of the quantum fingerprinting problem, we hope to provide results which will be easily tested by experiment, in order to help the development of implementations for more general quantum communication protocols.

It should be noted that Massar 7] proposed a single particle fingerprinting scheme, in which a particle is sent either to Alice or to Bob (in superposition). Each party induces a relative phase on the state of the particle, after which the two paths are interfered with one another. This scenario differs from the one considered here - for example, Alice and Bob share quantum information (the path taken by the particle) in addition to their input data $(\alpha$ and $\beta$ ), whereas no such additional information 
is available in the present scenario.

\section{PRELIMINARY DEFINITIONS}

Let $S$ be the set of possible strings that Alice and Bob may have. We consider cases where $|S|>2$ (cases with $|S| \leqslant 2$ are of course trivial to solve with one bit of communication).

Definition 1. Given the one (qu)bit messages from Alice and Bob, the referee will make a declaration (correct or incorrect) about whether or not their strings match.

- If the referee declares that the strings match, we say that his decision strategy accepts the input it was given, and we call this a positive result; if this declaration is incorrect, we may call this a false positive.

- If the referee declares that the strings differ, we say that his decision strategy rejects the input it was given, and we call this a negative result; if this declaration is incorrect, we may call this a false negative.

Definition 2. Here are some definitions related to error probabilities.

- For a given fingerprinting scheme, the error probability of the scheme will refer to the worst-case probability of an incorrect result in that scheme.

- A fingerprinting scheme with one-sided error is one where, if Alice and Bob have the same data string, the probability that the scheme will produce a false negative is zero.

\section{CLASSICAL ONE-BIT FINGERPRINTING}

In this section, we provide the definition of a one-bit fingerprinting scheme, and show that such schemes have quite limited power. Specifically, any one-bit fingerprinting scheme has an error probability of at least $\frac{1}{2}$ (which is the same error probability that results from just a fair coin flip by the referee). As well, any one-bit fingerprinting scheme with one-sided error has error probability 1 (the same error probability that results if the referee always declares that the strings match). Finally, any onebit fingerprinting scheme that succeeds with high probability when Alice's and Bob's strings match necessarily fails with high probability in $\Theta\left(|S|^{2}\right)$ of the cases where their strings do not match.

Definition 3. A one-bit fingerprinting scheme $\mathcal{P}$ operates as follows. To begin with, Alice and Bob receive inputs $\alpha, \beta \in S$, respectively. Each of them sends one bit to the referee, who produces an output bit based on these two messages. Each party may use a probabilistic strategy; however, there is no shared randomness among the parties. Such a scheme is characterised by three probability distributions:

- Alice's fingerprinting strategy $\left\{p_{\sigma}\right\}_{\sigma \in S}$, where $p_{\alpha}$ is the probability with which Alice sends the message $\mathbf{1}$, depending on her string $\alpha \in S$.

- Bob's fingerprinting strategy $\left\{q_{\sigma}\right\}_{\sigma \in S}$, where $q_{\beta}$ is the probability with which Bob sends the message $\mathbf{1}$, depending on his string $\beta \in S$.

- The referee's decision strategy $\left\{r_{00}, r_{01}, r_{10}, r_{11}\right\}$, where $r_{a b}$ is the probability with which the referee declares a positive result, depending on the bits $a$ and $b$ sent to him by Alice and Bob respectively.

Definition 4. For any one-bit fingerprinting scheme $\mathcal{P}$, define the function

$$
\begin{gathered}
\mathcal{P}_{+}(\alpha, \beta)=\left(1-p_{\alpha}\right)\left(1-q_{\beta}\right) r_{00}+\left(1-p_{\alpha}\right) q_{\beta} r_{01} \\
+p_{\alpha}\left(1-q_{\beta}\right) r_{10}+p_{\alpha} q_{\beta} r_{11} .
\end{gathered}
$$

That is, $\mathcal{P}_{+}(\alpha, \beta)$ is the probability that the scheme $\mathcal{P}$ yields a positive result when the input to Alice is $\alpha$ and to $\mathrm{Bob}$ is $\beta$.

We will usually want to place lower bounds on the probability of a positive result when $\alpha=\beta$. This will have a strong impact on the overall performance of the scheme $\mathcal{P}$. In order to simplify analysis, it will be useful to come up with a simple inequality.

Lemma 1. For any one-bit fingerprinting scheme $\mathcal{P}$, it is possible to define real-valued parameters $x_{\alpha}, y_{\beta}$, and $d_{\varepsilon}$ (depending on $\alpha, \beta \in S$ and $\varepsilon \in[0,1]$ respectively) such that

$$
x_{\alpha} y_{\beta} \geqslant d_{\varepsilon} \Longleftrightarrow \mathcal{P}_{+}(\alpha, \beta) \geqslant(1-\varepsilon) .
$$

Proof - Let $\alpha, \beta \in S$ be strings such that $\mathcal{P}_{+}(\alpha, \beta) \geqslant$ $(1-\varepsilon)$ holds. We may expand the expression of $\mathcal{P}_{+}(\alpha, \beta)$ in Equation 11 as a polynomial in $p_{\alpha}$ and $q_{\beta}$ : this gives us

$$
\begin{gathered}
\left(r_{00}-r_{01}-r_{10}+r_{11}\right) p_{\alpha} q_{\beta}+\left(r_{10}-r_{00}\right) p_{\alpha} \\
+\left(r_{01}-r_{00}\right) q_{\beta}+r_{00} \geqslant(1-\varepsilon) .
\end{gathered}
$$

We would like to separate the dependency on $\alpha$ and $\beta$ : how we may do this depends on the value of $c=\left(r_{00}-\right.$ $\left.r_{01}-r_{10}+r_{11}\right)$.

- If $c \neq 0$, we may define the parameters $x_{\alpha}, y_{\beta}$, and $d_{\varepsilon}$ as follows:

$$
\begin{aligned}
& x_{\alpha}=c\left(p_{\alpha}+\frac{r_{01}-r_{00}}{c}\right) \\
& y_{\beta}=q_{\beta}+\frac{r_{10}-r_{00}}{c} \\
& d_{\varepsilon}=(1-\varepsilon)+\frac{\left(r_{00}-r_{01}\right)\left(r_{00}-r_{10}\right)}{c}-r_{00} .
\end{aligned}
$$

Then, the inequality $x_{\alpha} y_{\beta} \geqslant d_{\varepsilon}$ holds, as it is equivalent to Equation 3 
- If $c=0$, we may drop the cross-term in $p_{\alpha}$ and $q_{\beta}$ from Equation 3 .

$$
\begin{aligned}
\left(r_{10}-r_{00}\right) p_{\alpha} & +\left(r_{01}-r_{00}\right) q_{\beta} \\
& +r_{00} \geqslant(1-\varepsilon) .
\end{aligned}
$$

As the exponential function $\left(x \longmapsto \mathrm{e}^{x}\right)$ is monotone increasing, the inequality will be preserved if we exponentiate both sides. Doing this yields

$$
\mathrm{e}^{\left(r_{10}-r_{00}\right) p_{\alpha}} \mathrm{e}^{\left(r_{01}-r_{00}\right) q_{\beta}} \mathrm{e}^{r_{00}} \geqslant \mathrm{e}^{1-\varepsilon} .
$$

In this case, we may define the parameters $x_{\alpha}, y_{\beta}$, and $d_{\varepsilon}$ as follows:

$$
\begin{aligned}
& x_{\alpha}=\mathrm{e}^{\left(r_{10}-r_{00}\right) p_{\alpha}} \\
& y_{\beta}=\mathrm{e}^{\left(r_{01}-r_{00}\right) q_{\beta}} \\
& d_{\varepsilon}=\mathrm{e}^{1-\varepsilon-r_{00}} .
\end{aligned}
$$

Then, we have the inequality $x_{\alpha} y_{\beta} \geqslant d_{\varepsilon}$.

In both cases, the parameters are defined so that if $\mathcal{P}(\alpha, \beta) \geqslant(1-\varepsilon)$, the inequality $x_{\alpha} y_{\beta} \geqslant d_{\varepsilon}$ holds. For the converse, suppose that $x_{\alpha} y_{\beta} \geqslant d_{\varepsilon}$ for two particular strings $\alpha, \beta \in S$. By simply applying the definitions of $x_{\alpha}, y_{\beta}$, and $d_{\varepsilon}$ (and taking the logarithms of both sides if $c=0$ ), we can immediately recover Equation 3 Thus, the converse holds as well.

The parameters $x_{\alpha}, y_{\beta}$, and $d_{\varepsilon}$ do not necessarily refer to any obvious properties of the scheme $\mathcal{P}$. However, using them, we may easily prove very restrictive upper bounds on the effectiveness of one-bit fingerprinting schemes:

Theorem 1. Let $\mathcal{P}$ be a one-bit fingerprinting scheme. If $\varepsilon$ is an upper bound for the worst-case probability that $\mathcal{P}$ produces false negatives, then $(1-\varepsilon)$ is a lower bound on the worst-case probability that $\mathcal{P}$ will produce false positives.

Proof - Because the probability of a false negative is at most $\varepsilon$, we have $\mathcal{P}_{+}(\sigma, \sigma) \geqslant(1-\varepsilon)$ for all strings $\sigma \in S$. Then, by Lemma [1 we also have $x_{\sigma} y_{\sigma} \geqslant d_{\varepsilon}$ for all $\sigma \in S$. Consider an alternative definition of the 'sign' function:

$$
\operatorname{sgn} x_{\sigma}=\left\{\begin{array}{rl}
1, & x_{\sigma} \geqslant 0 \\
-1, & x_{\sigma}<0
\end{array} .\right.
$$

As there are two possible values for the 'sign' function, and more than two elements in $S$, there must then be at least two distinct strings $\mu, \nu \in S$ such that $\operatorname{sgn} x_{\mu}=$ $\operatorname{sgn} x_{\nu}$. Without loss of generality, we may choose $\mu$ and $\nu$ such that $\left(\operatorname{sgn} x_{\nu}\right) y_{\nu} \geqslant\left(\operatorname{sgn} x_{\mu}\right) y_{\mu}$. Then we have

$$
x_{\mu} y_{\nu} \geqslant x_{\mu} y_{\mu} \geqslant d_{\varepsilon} .
$$

Again by Lemma 1 we then know that $\mathcal{P}_{+}(\mu, \nu) \geqslant$ $(1-\varepsilon)$. As $\mu$ and $\nu$ were chosen to be distinct, the worstcase probability of a false positive for the fingerprinting scheme $\mathcal{P}$ is then at least $(1-\varepsilon)$.
Corollary 1-1. Any one-bit fingerprinting scheme has error probability at least $\frac{1}{2}$.

Corollary 1-2. Any one-sided error one-bit fingerprinting scheme has error probability 1.

Not only are there lower bounds on the worst-case probability of failure, but there is also a lower bound on the number of inputs which are likely to produce a false positive:

Theorem 2. Let $\mathcal{P}$ be a one-bit fingerprinting scheme, and let $|S|=s$. If the worst-case probability of obtaining a false negative is at most $\varepsilon \geqslant 0$ in $\mathcal{P}$, then the number of inputs $(\alpha, \beta) \in S \times S$ such that $\alpha \neq \beta$ and $\mathcal{P}_{+}(\alpha, \beta) \geqslant$ $(1-\varepsilon)$ is at least $\frac{1}{4}\left(s^{2}-2 s\right)$.

Proof sketch - This follows by considering the arguments from Theorem 1] We may establish a lower bound by counting the number of unordered pairs $\{\alpha, \beta\}$ such that $\operatorname{sgn} x_{\alpha}=\operatorname{sgn} x_{\beta}$ and $\alpha \neq \beta$. This is minimized if nearly half of the strings $\sigma \in S$ have $\operatorname{sgn} x_{\sigma}=1$, and nearly half again have $\operatorname{sgn} x_{\sigma}=-1$. If this is the case, a simple counting argument shows that the number of such (unordered) pairs is $2\left(\begin{array}{c}s / 2 \\ 2\end{array}\right)=\frac{1}{4}\left(s^{2}-2 s\right)$ for $s$ even, and $\left(\begin{array}{c}(s+1) / 2 \\ 2\end{array}\right)+\left(\begin{array}{c}(s-1) / 2 \\ 2\end{array}\right)=\frac{1}{4}\left(s^{2}-2 s+1\right)$ for $s$ odd.

So, one is forced to accept one of two scenarios in a onebit fingerprinting scheme: one must either forego a reasonable upper bound on the probability of error when Alice's and Bob's strings match, or use a scheme which will produce false positives with high probability for about $\frac{1}{4}$ or more of Alice's and Bob's possible inputs (strictly more than the number of matching cases when $|S|>6$ ).

\section{QUANTUM ONE-QUBIT FINGERPRINTING}

In this section, we provide the definition of a onequbit fingerprinting scheme, give examples of one-qubit schemes that outperform what classical one-bit fingerprinting schemes can achieve, and give some upper and lower bounds for the performance of schemes with onesided error.

Definition 5. A one-qubit fingerprinting scheme $\mathcal{Q}$ operates as follows. To begin with, Alice and Bob receive inputs $\alpha, \beta \in S$, respectively. Then each of them sends one qubit to the referee, who produces an output bit based on these two qubits. There is no a priori shared information among the parties. Such a scheme is characterized by three components:

- Alice's message set $\left\{\rho_{\sigma}\right\}_{\sigma \in S}$, where $\rho_{\alpha}$ is the onequbit (possibly mixed) state that Alice will prepare and send to the referee, depending on her string $\alpha$.

- Bob's message set $\left\{\tau_{\sigma}\right\}_{\sigma \in S}$, where $\tau_{\beta}$ is the onequbit (possibly mixed) state that Bob will prepare and send to the referee, depending on his string $\beta$. 
- The referee's decision strategy, which is some twooutcome measurement of the two-qubit state $\rho_{\alpha} \otimes$ $\tau_{\beta}$, possibly using some finite number of ancilliary qubits.

The inputs recieved by the referee fall into two classes: matching states, which are states of the form $\rho_{\sigma} \otimes \tau_{\sigma}$ for some $\sigma \in S$, and hybrid states, which are states of the form $\rho_{\alpha} \otimes \tau_{\beta}$ for $\alpha \neq \beta$. The goal of the referee is essentially to attempt to distinguish matching states from hybrid states.

\section{A. Some one-qubit fingerprinting schemes}

In this section, we give examples of one-qubit fingerprinting schemes that outperform any possible classical one-bit scheme.

The basic framework that we use for constructing fingerprinting schemes is that of [3]: construct a set of pure fingerprint states with small pairwise inner product and then apply a controlled-SWAP test.

Theorem 3. Let $\mathcal{Q}$ be a fingerprinting scheme with the following characteristics:

- Alice and Bob both use the same set of pure states $\left\{\left|\phi_{\sigma}\right\rangle\right\}_{\sigma \in S}$ as fingerprints, such that for all $\alpha, \beta \in$ $S$ with $\alpha \neq \beta,\left|\left\langle\phi_{\alpha} \mid \phi_{\beta}\right\rangle\right| \leqslant \delta$;

- The referee's decision strategy is to compute the state

$$
(H \otimes I \otimes I) \mathrm{C}-\operatorname{SWAP}(H \otimes I \otimes I)|0\rangle\left|\phi_{\alpha}\right\rangle\left|\phi_{\beta}\right\rangle,
$$

measure the first qubit, and declare a negative result iff the result is $\mathbf{1}$.

Then $\mathcal{Q}$ is a one-sided error fingerprinting scheme, with error probability at most $\frac{1+\delta^{2}}{2}$.

Using this framework for one-qubit fingerprinting schemes, the task is to find sets of one-qubit fingerprint states such that their pairwise inner product is as small as possible. Using the Bloch sphere representation for qubit states, this reduces to finding points on the Bloch sphere which are as widely separated as possible. For instance, for $|S|=4$, one would use the vertices of a regular tetrahedron inscribed within the Bloch sphere, such as

$$
\begin{aligned}
& \left|\phi_{0}\right\rangle=|0\rangle \\
& \left|\phi_{1}\right\rangle=\frac{1}{\sqrt{3}}(|0\rangle+\sqrt{2}|1\rangle) \\
& \left|\phi_{2}\right\rangle=\frac{1}{\sqrt{3}}\left(|0\rangle+\mathrm{e}^{2 \pi i / 3} \sqrt{2}|1\rangle\right) \\
& \left|\phi_{3}\right\rangle=\frac{1}{\sqrt{3}}\left(|0\rangle+\mathrm{e}^{4 \pi i / 3} \sqrt{2}|1\rangle\right) .
\end{aligned}
$$

The inner product of any two of these states is $\frac{1}{\sqrt{3}}$ in absolute value. Similarly, for $|S|=3$ one would use the vertices of an equilateral triangle, and for $|S|=6$ one would use the vertices of a regular octahedron. The (maximal) absolute value of the inner product of two states are $\frac{1}{2}$ and $\frac{1}{\sqrt{2}}$, respectively, in the latter two cases. For $|S|=s \gg 1$, there exists a collection of $s$ states whose maximal pairwise inner product is $1-O\left(\frac{1}{s}\right)$ in absolute value (this may be shown using a simple geometric argument, by considering the area available to be used in distributing the states on the Bloch sphere).

Using these figures for the magnitude of the inner product in various cases, we may use Theorem 3 to construct fingerprinting schemes with one sided error, each of which outperforms any possible one-bit fingerprinting scheme with one-sided error. The error probabilities for these schemes are presented in Table $\llbracket$ below.

Any one-sided error fingerprinting scheme with error probability $\varepsilon$ can be converted into a general (two-sided error) scheme with error probability $\frac{\varepsilon}{1+\varepsilon}$, as follows. After running the one-sided error scheme, if the result is positive, we change the result to negative with probability $\frac{\varepsilon}{1+\varepsilon}$. It is straightforward to verify that the resulting (two-sided) error probability will be $\frac{\varepsilon}{1+\varepsilon}$. Using this observation, we can determine the error probabilties for one-qubit fingerprinting schemes with two-sided error, each of which again outperforms any possible one-bit fingerprinting scheme with two-sided error. These error probabilities are also presented in Table [

\begin{tabular}{|c|c|c|c|}
\hline$|S|$ & $\begin{array}{c}\text { Maximal } \\
\text { inner product }\end{array}$ & $\begin{array}{c}\text { Error probability } \\
(1 \text {-sided error })\end{array}$ & $\begin{array}{c}\text { Error probability } \\
(2 \text {-sided error })\end{array}$ \\
\hline 3 & $\frac{1}{2}$ & $\frac{5}{8}=0.625$ & $\frac{5}{13} \approx 0.385$ \\
4 & $\frac{1}{\sqrt{3}}$ & $\frac{2}{3} \approx 0.667$ & $\frac{2}{5}=0.400$ \\
6 & $\frac{1}{\sqrt{2}}$ & $\frac{3}{4}=0.750$ & $\frac{3}{7} \approx 0.429$ \\
$s \gg 1$ & $1-O\left(\frac{1}{s}\right)$ & $1-O\left(\frac{1}{s}\right)$ & $\frac{1}{2}-O\left(\frac{1}{s}\right)$ \\
\hline
\end{tabular}

TABLE I: For $|S|=3,4,6$, and the asymptotic case $s \gg$ 1 , the error probabilities for some one-qubit fingerprinting schemes in the framework of [3].

Note that the one-sided error probabilities are all strictly less than 1, and the two-sided error probabilities are all strictly less than $\frac{1}{2}$, neither of which are possible for one-bit fingerprinting schemes (by Corollary 1-2] and Corollary 1-1 respectively).

\section{B. Error probabilities in one-qubit fingerprinting schemes}

In this section, we give some upper and lower bounds on the error probabilities of one-qubit fingerprinting schemes with one-sided error. In doing so, we obtain results which imply that when Alice's choice of fingerprint states is evenly distributed on the Bloch sphere, the optimal one-qubit scheme is symmetric (that is, where Alice and Bob use the same fingerprint states). We also show that there are choices of fingerprint states for Alice 
where a symmetric scheme is not optimal, and discuss necessary conditions which must hold for a symmetric scheme to be a sub-optimal choice. These results suggest that for even such a small instance of quantum fingerprinting, the question of finding an optimal scheme for a fixed value $|S|$ is not simple: in particular, analysis in the general case does not lend itself to simple arguments by symmetry.

It will be useful to define some additional notation. Throughout this section, for a one-qubit fingerprinting scheme $\mathcal{Q}$, we represent the worst-case probability of a false negative by $W_{\mathcal{Q}}^{-}$, and the worst-case probability of a false positive by $W_{\mathcal{Q}}^{+}$.

\section{One-qubit fingerprinting with one-sided error}

As we saw in section III a one-bit fingerprinting scheme with one-sided error will have an error probability of 1. Because we have no interest in one-qubit schemes with this error probability, we will use the following terminology:

Definition 6. A fingerprinting scheme is strict if it has one-sided error $\left(W_{\mathcal{Q}}^{-}=0\right)$ and an error probability strictly less than $1\left(W_{\mathcal{Q}}^{+}<1\right)$.

In a strict one-qubit fingerprinting scheme $\mathcal{Q}$, the referee's decision strategy must accept any matching state $\rho_{\sigma} \otimes \tau_{\sigma}$ (for $\sigma \in S$ ). This leads to a natural definition:

Definition 7. The accept space $A_{\mathcal{Q}}$ of a one-qubit fingerprinting scheme $\mathcal{Q}$ is the set of two-qubit pure states $|\gamma\rangle$, such that the referee's decision strategy would produce a positive result with certainty if it were provided the input $|\gamma\rangle$ (regardless of whether $|\gamma\rangle$ is a product of fingerprint states from Alice and Bob).

The accept space is a vector space: if the referee's strategy accepts two distinct two-qubit states $|\gamma\rangle$ and $\left|\gamma^{\prime}\right\rangle$ with certainty, by linearity it must accept any linear combination of $|\gamma\rangle$ and $\left|\gamma^{\prime}\right\rangle$.

In order to be accepted with certainty, each matching state must be a (possibly trivial) mixture of states in the accept space. So, it is natural to try to understand a fingerprinting scheme $\mathcal{Q}$ in terms of $A_{\mathcal{Q}}$. One question which bears interest is how large the accept space can be for a strict one-qubit fingerprinting scheme.

Theorem 4. If $\mathcal{Q}$ is a strict one-qubit fingerprinting scheme, then $\operatorname{dim} A_{\mathcal{Q}}=3$.

Proof sketch - First, it is useful to note that neither Alice nor Bob can use one fingerprint state for two distinct data strings: this would lead immediately to the existence of a matching state (which must be accepted with certainty) which is identical with some hybrid state (which must not be accepted with certainty). So, each of Alice's fingerprint states must be distinct from one another, and similarly for Bob. We may then prove the theorem by eliminating every other possible value of $\operatorname{dim} A_{\mathcal{Q}}$.
The fact that both Alice and Bob must use distinct fingerprints for distinct input strings implies $\operatorname{dim} A_{\mathcal{Q}}>1$. Using arguments of linear independence, it is possible to show that if Alice or Bob use mixed states for fingerprints, then $\operatorname{dim} A_{\mathcal{Q}}>2$. On the other hand, if Alice uses the pure states $\left|\phi_{\sigma}\right\rangle$ and Bob uses the pure states $\left|\psi_{\sigma}\right\rangle$ for $\sigma \in S$, we can determine that $\operatorname{dim} A_{\mathcal{Q}}>2$ from the fact that the equation

$$
\left|\phi_{\omega}\right\rangle\left|\psi_{\omega}\right\rangle=c_{1}\left|\phi_{\mu}\right\rangle\left|\psi_{\mu}\right\rangle+c_{2}\left|\phi_{\nu}\right\rangle\left|\psi_{\nu}\right\rangle
$$

cannot be satisfied for any $c_{1}, c_{2} \in \mathbb{C}$ for any three strings $\mu, \nu, \omega \in S$ (the state on the left-hand side being both a product state and linearly independent from both of the states on the right-hand side individually). Finally, because there must be a non-zero probability of rejecting hybrid states, we must have $\operatorname{dim} A_{\mathcal{Q}}<4$. Thus we have $\operatorname{dim} A_{\mathcal{Q}}=3$, as required.

This restriction on the nature of $A_{\mathcal{Q}}$ has some strong implications about what states Alice and Bob may use for fingerprints. The simplest is as follows:

Theorem 5. In a strict one-qubit fingerprinting scheme, Alice and Bob must use pure states for fingerprint states.

This result is easy to demonstrate: the proof can be found in the appendix. On the other hand, this result is not clearly trivial — without this theorem, there is no immediately obvious reason to exclude mixed quantum fingerprinting schemes, even for reasons of optimality. (In the classical scheme where we allow arbitrary length fingerprints, 8] provides a mixed fingerprinting strategy which provides optimal performance for a classical fingerprinting scheme.) In consideration of Theorem [5] we will use the pure state $\left|\phi_{\sigma}\right\rangle$ to describe Alice's fingerprint for $\sigma \in S$, and $\left|\psi_{\sigma}\right\rangle$ to describe Bob's fingerprints, for $\sigma \in S$, in any strict one-qubit fingerprinting scheme.

For a strict scheme $\mathcal{Q}$, we may consider the set of twoqubit states which are perfectly distinguishable from the matching states. Because the accept space defined by the matching states is of dimension 3, there will be precisely one such state, which we denote by $\left|R_{\mathcal{Q}}\right\rangle$. Being orthogonal to the accept space, it is the two-qubit state which the referee's decision strategy would be most likely to reject. As a result, we call $\left|R_{\mathcal{Q}}\right\rangle$ the reject state of $\mathcal{Q}$. Although it is possible for the referee's strategy to accept the state $\left|R_{\mathcal{Q}}\right\rangle$ with non-zero probability, an optimal choice of decision strategy for the referee is to reject $\left|R_{\mathcal{Q}}\right\rangle$ with certainty.

Theorem 6. Let $C$ be a (non-empty) collection of strict one-qubit fingerprinting schemes, characterized by some choice of fingerprint states $\left\{\left|\phi_{\sigma}\right\rangle\right\}_{\sigma \in S}$ for Alice and $\left\{\left|\psi_{\sigma}\right\rangle\right\}_{\sigma \in S}$ for Bob. Let $A$ be the accept space defined by these fingerprint states, and $|R\rangle$ the corresponding reject state. Then for any $\mathcal{Q} \in C$,

$$
W_{\mathcal{Q}}^{+} \geqslant 1-\min _{\alpha \neq \beta} \mid\left\langle R\left|\left(\left|\phi_{\alpha}\right\rangle \otimes\left|\psi_{\beta}\right\rangle\right)\right|^{2},\right.
$$


with equality when the referee's decision strategy is to project $\left|\phi_{\alpha}\right\rangle\left|\psi_{\beta}\right\rangle$ either into $A$ or onto $|R\rangle$ using a measurement, returning a negative result iff the result of this measurement is $|R\rangle$.

Proof - That the strategy described above yields a strict one-qubit fingerprinting scheme is trivial: we will show that this is the optimal decision strategy for the referee. Whatever the referee's decision strategy may be, it must attempt to distinguish between the hybrid states and the accept space without having specific knowledge about the input he receives. Any such strategy can be described by a two-valued POVM $\left\{E_{+}, E_{-}\right\}$, where $E_{+}$ and $E_{-}$corresponds positive and negative results respectively. For any state $|\psi\rangle \in A$, we require

$$
\operatorname{tr}\left(E_{-}|\psi\rangle\langle\psi|\right)=0,
$$

which implies $E_{-}=p|R\rangle\langle R|$ for some probability $p \geqslant 0$. Then, the POVM which describes the referee's strategy is equivalent to a partial projective measurement (either onto the the state $|R\rangle$ or into the space $A$ ), followed by a biased coin-flip to decide whether to produce a negative result should the state collapse to $|R\rangle$. In order to achieve the greatest probability of distinguishing hybrid states from the accept space, we may choose to fix $p=1$. The strategy that we obtain is then precisely the one described by the theorem.

The reject state can be a useful tool for analysis. For instance, consider the Schmidt decomposition of $\left|R_{\mathcal{Q}}\right\rangle$, which can be expressed as

$$
\left|R_{\mathcal{Q}}\right\rangle=\frac{1}{\sqrt{1+C^{2}}}\left(\left|\eta_{0}\right\rangle\left|\kappa_{0}\right\rangle-C\left|\eta_{1}\right\rangle\left|\kappa_{1}\right\rangle\right)
$$

for some orthonormal bases $\left\{\left|\eta_{0}\right\rangle,\left|\eta_{1}\right\rangle\right\}$ and $\left\{\left|\kappa_{0}\right\rangle,\left|\kappa_{1}\right\rangle\right\}$ of $\mathbb{C}^{2}$, and some real coefficient $C>0$. (Because Alice and Bob are not allowed to use the same fingerprint state for different strings, it is simple to show that $C \neq 0$, and we may absorb any relative phase into the definitions of $\left|\eta_{1}\right\rangle$ and $\left|\kappa_{1}\right\rangle$.) Let $U=|0\rangle\left\langle\eta_{0}|+| 1\right\rangle\left\langle\eta_{1}\right|$ and $V=|1\rangle\left\langle\kappa_{0}|+| 0\right\rangle\left\langle\kappa_{1}\right|$. Then, we have

$$
(U \otimes V)\left|R_{\mathcal{Q}}\right\rangle=\frac{1}{\sqrt{1+C^{2}}}(|01\rangle-C|10\rangle)
$$

As a part of his decision strategy, the referee can perform the transformation $U \otimes V$ as the first step, without loss of generality. However, because $U \otimes V$ can also be carried out without communication by Alice and Bob as the last part of the preparation of their fingerprint states, $\mathcal{Q}$ is equivalent to a different one-qubit scheme $\mathcal{Q}^{\prime}$, where Alice and Bob use the fingerprints $U\left|\phi_{\sigma}\right\rangle$ and $V\left|\psi_{\sigma}\right\rangle$ (respectively) for the string $\sigma \in S$ rather than $\left|\phi_{\sigma}\right\rangle$ and $\left|\psi_{\sigma}\right\rangle$, and where the reject state is $(U \otimes V)\left|R_{\mathcal{Q}}\right\rangle$ rather than $\left|R_{\mathcal{Q}}\right\rangle$. For the sake of brevity, we will call a strict scheme $\mathcal{Q}$ canonical if the reject state is given by $\left|R_{\mathcal{Q}}\right\rangle=\frac{1}{\sqrt{1+C^{2}}}(|01\rangle-C|10\rangle)$ for some $C>0$. For any strict scheme $\mathcal{Q}$, we will call the canonical fingerprinting scheme which is equivalent to $\mathcal{Q}$ the canonical form of $\mathcal{Q}$.

Given the canonical form of $\mathcal{Q}$, we can easily describe a relation between Alice's and Bob's fingerprint states. We can express $\left|\phi_{\sigma}\right\rangle$ and $\left|\psi_{\sigma}\right\rangle$ in the form

$$
\begin{aligned}
& \left|\phi_{\sigma}\right\rangle=\frac{1}{\sqrt{1+\left|u_{\sigma}\right|^{2}}}\left(|0\rangle+u_{\sigma}|1\rangle\right) \\
& \left|\psi_{\sigma}\right\rangle=\frac{1}{\sqrt{1+\left|w_{\sigma}\right|^{2}}}\left(|0\rangle+w_{\sigma}|1\rangle\right),
\end{aligned}
$$

for some complex constants $u_{s}, w_{s} \in \mathbb{C}$. (For $\left|\phi_{\sigma}\right\rangle=$ $|1\rangle$, we may take the limit $\left|u_{\sigma}\right| \rightarrow \infty$, and similarly for $\left.\left|\psi_{\sigma}\right\rangle.\right)^{1}$ Using the expression in Equation 14 for $\left|R_{\mathcal{Q}}\right\rangle$, from $\left\langle R_{\mathcal{Q}}\left|\left(\left|\phi_{\sigma}\right\rangle \otimes\left|\psi_{\sigma}\right\rangle\right)=0\right.\right.$, we can then determine

$$
\begin{gathered}
w_{\sigma}\langle 01 \mid 01\rangle-C u_{\sigma}\langle 10 \mid 10\rangle=0 \\
\Longrightarrow w_{\sigma}=C u_{\sigma} .
\end{gathered}
$$

(This equation extends appropriately in the limit $\left|u_{\sigma}\right|,\left|w_{\sigma}\right| \rightarrow \infty$ in the sense that $\left|\phi_{\sigma}\right\rangle\left|\psi_{\sigma}\right\rangle$ will approach $|11\rangle$, which is an element of $A_{\mathcal{Q}}$.) Then, if the reject state $\left|R_{\mathcal{Q}}\right\rangle$ of a strict one-qubit scheme $\mathcal{Q}$ is known, one can determine Bob's fingerprints from Alice's fingerprints, using the canonical form of $\mathcal{Q}$. One can then characterize a strict one-qubit fingerprinting protocol by its' reject state $\left|R_{\mathcal{Q}}\right\rangle$, the probability with which the referee rejects $\left|R_{\mathcal{Q}}\right\rangle$, and Alice's choice of fingerprint states.

Using this observation, we can also determine useful information about a strict one-qubit scheme from only Alice's or only Bob's fingerprint states, along with the parameter $C$ used to define the reject state.

Theorem 7. Let $\mathcal{Q}$ be a strict one-qubit fingerprinting scheme, where Alice's fingerprint states are given by $\left\{\left|\phi_{\sigma}\right\rangle\right\}_{\sigma \in S}$ and Bob's fingerprint states are given by $\left\{\left|\psi_{\sigma}\right\rangle\right\}_{\sigma \in S}$. For the sake of brevity, define the hybrid state $\left|h_{\alpha \beta}\right\rangle=\left|\phi_{\alpha}\right\rangle\left|\psi_{\beta}\right\rangle$ for each $\alpha \neq \beta$. Then, there is a collection of positive real constants $\left\{K_{\sigma}\right\}_{\sigma \in S}$ such that

$$
\left|\left\langle R_{\mathcal{Q}} \mid h_{\alpha \beta}\right\rangle\right|^{2}=K_{\beta}\left(1-\left|\left\langle\phi_{\beta} \mid \phi_{\alpha}\right\rangle\right|^{2}\right),
$$

for all $\alpha, \beta \in S$.

With this result, knowledge of the constants $K_{\sigma}$ for $\sigma \in S$ allows one to easily determine the probability that any hybrid state will be projected onto $\left|R_{\mathcal{Q}}\right\rangle$, using only Alice's fingerprints. The proof of Theorem[7] is left to the appendix; for schemes $\mathcal{Q}$ in canonical form, the parameter $K_{\sigma}$ can be given as a function of $C$, and the extended real number $u_{\sigma}$ which defines Alice's state $\left|\phi_{\sigma}\right\rangle$ :

$$
K_{\sigma}=\frac{C^{2}\left(1+\left|u_{\sigma}\right|^{2}\right)}{\left(1+C^{2}\left|u_{\sigma}\right|^{2}\right)\left(1+C^{2}\right)}
$$

\footnotetext{
1 To ensure that this is meaningful, we take care to observe that expressions involving $\left|u_{\sigma}\right|$ and $\left|w_{\sigma}\right|$ extend in an appropriate way under this limit.
} 
(As $\left|u_{\sigma}\right| \rightarrow \infty$, this formula converges to $\frac{1}{1+C^{2}}$.) It is easy to show that, as a function of $\left|u_{\sigma}\right|, K_{\sigma}$ is constant for $C=1$, monotone increasing for $C<1$, and monotone decreasing for $C>1$. Of particular interest is the locus of possible values of $u_{\sigma}$ where $K_{\sigma}=\frac{1}{2}$ : it is simple to show that for any value of $C$, this set of points will be the circle of radius $C^{-1}$ about the origin.

By considering this formula for $K_{\sigma}$, we can begin to try finding an optimal strict fingerprinting scheme (by optimising the value of $C$ ) based on Alice's fingerprint states, and vice-versa. We will describe some ideas of how this may be done in the following section.

\section{Strict symmetric fingerprinting schemes}

Fingerprinting schemes where Alice and Bob use the same states as each other for their fingerprints are a natural class of fingerprinting schemes to consider. All of the results obtained in the previous section hold for the special case of strict symmetric one-qubit fingerprinting schemes: in this section, we will elaborate on some of the results for this special case, and discuss when a symmetric scheme is an optimal choice of one-qubit fingerprinting scheme.

It is easy to verify that, for a symmetric scheme, $\left|R_{\mathcal{Q}}\right\rangle=\left|\Psi^{-}\right\rangle$. Theorem [6] then effectively reproduces the controlled-sWAP decision strategy of Theorem [3 as $\left|\Psi^{-}\right\rangle$is a -1 eigenstate of the SWAP operation, and the other eigenstates of SWAP have the eigenvalue +1 . As well, $\left|R_{\mathcal{Q}}\right\rangle=\left|\Psi^{-}\right\rangle$gives us $C=1$ for $\mathcal{Q}$, which implies that $K_{\sigma}=\frac{1}{2}$ for all $\sigma \in S$. Theorem $[7$ then simplifies to

$$
\left|\left\langle R_{\mathcal{Q}} \mid h_{\alpha \beta}\right\rangle\right|^{2}=\frac{1-\left|\left\langle\phi_{\beta} \mid \phi_{\alpha}\right\rangle\right|^{2}}{2} .
$$

The probability of rejection obtained is then consistent with the results of [3].

Considering $K_{\sigma}$ as a function of $C$ and $u_{\sigma}$, the value $C=1$ is the only value for which $K_{\sigma}$ is not strictly increasing or strictly decreasing. As a result, the choice $C=1$ plays a somewhat special role in the analysis of strict one-qubit schemes. One approach to examining one-qubit fingerprinting schemes is to fix Alice's distribution of fingerprints, and try to determine which value of $C$ leads to the best error probability for the scheme as a whole. Under some reasonable assumptions on Alice's choice of states, the value $C=1$ (and thus, a symmetric scheme) is optimal. However, this is not true under more general assumptions.

Suppose Alice has some fixed choice of fingerprinting states. Among Alice's fingerprints, there will be some pair of states $\left|\phi_{\alpha}\right\rangle,\left|\phi_{\beta}\right\rangle$ which have the largest inner product of any pair. For one-qubit schemes in canonical form, in order for an asymmetric scheme to perform better than a symmetric scheme, we require $K_{\alpha}, K_{\beta}>\frac{1}{2}$ for this pair of $\alpha$ and $\beta$. This is possible only if both $u_{\alpha}$ and $u_{\beta}$ lie within a circle of radius $C^{-1}$ about the origin on the complex plane for $C>1$, or outside such a circle for $C<1$. Either of these constraints on the values of $u_{\alpha}$ and $u_{\beta}$ is equivalent to the states $\left|\phi_{\alpha}\right\rangle$ and $\left|\phi_{\beta}\right\rangle$ lying within some circle of lattitude, either closer to $|0\rangle$ or to $|1\rangle$, on the Bloch sphere. Then, the closer such a minimally distinguishable pair lies to the equator of the Bloch sphere, the closer $C$ must be to 1 for these criteria to apply, and so the closer the optimal scheme will be to symmetric.

Using this observation, we can determine some necessary criteria on Alice's choice of fingerprints, in order for a symmetric scheme to be suboptimal for that choice. For a minimally distinguishable pair of states $\left|\phi_{\alpha}\right\rangle$ and $\left|\phi_{\beta}\right\rangle$ of Alice's, if

- one of the pair lies on the equator of the Bloch sphere,

- the two states lie on opposite sides of the Bloch equator, or

- two such pairs of minimal distinguishability lie on opposite sides of the Bloch equator,

then it is easy to show that a symmetric protocol is optimal. (In particular, for a scheme where Alice's states are optimally spaced on the Bloch sphere, the latter two conditions will most likely apply.) In order for $C>1$ to be optimal, we then require $u_{\alpha}$ and $u_{\beta}$ to both lie within a circle of radius $C^{-1}$ on the complex plane, and for $\left|\left\langle\phi_{\alpha} \mid \phi_{\beta}\right\rangle\right|$ to be strictly larger than the inner product of any other pair. For $C<1$ to be optimal, we have similar requirements, except that $u_{\alpha}$ and $u_{\beta}$ must both lie outside the circle of radius $C^{-1}$ on the complex plane.

It is easy to find simple choices of fingerprint states for Alice where a symmetric scheme is not optimal. One example would be where $S=\{0,1,2\}$, and we define Alice's states by the complex parameters

$$
u_{0}=0 ; \quad u_{1}=2 ; \quad u_{2}=-2 .
$$

In this case, the minimally distinguishable pair would be $\left|\phi_{1}\right\rangle$ and $\left|\phi_{2}\right\rangle$ with an inner product of $\frac{3}{5}$, compared to $\frac{1}{5}$ for the other two possible pairs. Using Theorem 7 with the value $K_{\sigma}=\frac{1}{2}$ for all $\sigma \in S$, a symmetric scheme $\mathcal{Q}$ with this choice of fingerprints for Alice would have error probability $\frac{17}{25}=0.68$. However, the points $u_{1}$ and $u_{2}$ both lie outside the circle of radius $C^{-1}$ on the complex plane, for any $\frac{1}{2}<C<1$. In particular, consider a scheme $\mathcal{Q}_{A}$ where $C=\frac{1}{\sqrt{2}}$. This choice of $C$ will yield

$$
K_{0}=\frac{1}{3} ; \quad K_{1}=K_{2}=\frac{5}{9}
$$

The higher values for $K_{1}$ and $K_{2}$ improve the probability of error in the worst case at the cost of the probability of success in other cases. Using Theorem 7 once more, we may determine the probability of error when $\alpha=1$ and $\beta=2$ to be $\frac{29}{45} \approx 0.644$. It is easy to determine that this is the error probability of $\mathcal{Q}_{A}$. Although this is not an improvement on the error probability of the strict 
protocol for $|S|=3$ presented in Table \ this is better than in the symmetric protocol $\mathcal{Q}_{S}$. Then, for this choice of fingerprint states for Alice, the scheme $\mathcal{Q}_{A}$ has a better worst-case probability of error than a symmetric scheme.

In general, evenly spacing Alice's states on the Bloch sphere will lead to a symmetric fingerprinting scheme being the optimal choice, because there will not be a unique pair with maximal inner product, and the distribution of minimally distinguishable pairs will be essentially symmetric on the Bloch sphere. However, for an arbitrary distribution of Alice's states, a symmetric protocol is not optimal, and it appears difficult to show that evenly spacing states on the Bloch sphere is an optimal choice without first assuming a symmetric scheme is to be used.

\section{CONCLUSION}

We have shown that fingerprinting schemes that make use of only one-qubit messages from Alice and Bob to the referee can perform better in the worst case than is possible for any similar classical communication scheme using single bit messages. This result holds whether one requires one-sided error (with no false negatives) or one allows two-sided error probabilities.

For a one-bit fingerprinting scheme, one can never achieve a better worst-case performance than blind guessing, and one-sided error schemes have error probability 1. The number of inputs for Alice and Bob where the fingerprinting scheme performs "badly" (with a high lower bound on the error probability) either includes all inputs where Alice's and Bob's string match, or a sizeable fraction of the other possible inputs.

In the quantum case, while some lower bounds exist, upper bounds for error probability can also be easily derived for one-qubit fingerprinting schemes with one-sided error, and can be determined from the fingerprint states of either Alice or Bob alone if the reject state is known. Using these techniques, it is possible to show that there are choices of fingerprints for Alice, for which the optimal one-qubit scheme is not symmetric. However, if it is assumed that Alice distributes her states as evenly over the Bloch sphere as possible, a symmetric scheme is quite likely the optimal scheme.

It is more difficult to make clear statements one-qubit fingerprinting schemes with two-sided error. Although it is easy to find ones with reasonably low error probability by converting one-sided error schemes, the structure of strict schemes is lost: the freedom to reject matching states with some probability makes simple arguments by linearity difficult to find.

\section{Acknowledgements}

I would like to thank Richard Cleve for suggesting this problem, providing initial insights, and useful critical advice on this article.

\section{APPENDIX: DEFERRED PROOFS}

We will now present the two results from the analysis of strict one-qubit fingerprinting schemes whose proofs were deferred.

To prove Theorem [5 we will use a number of ideas introduced originally after the theorem: namely, the idea of the reject state (defined on page 5), and the idea of the canonical form of a strict protocol $\mathcal{Q}$ (presented on page (6). These two ideas do not depend on Alice's and Bob's fingerprint states being pure states. We would also like to use the linear relation of Equation [16] which we will briefly re-derive here.

Proof of Theorem [5 - Without loss of generality, $\mathcal{Q}$ is in canonical form. Then, let $C$ be a positive real such that

$$
\left|R_{\mathcal{Q}}\right\rangle=\frac{1}{\sqrt{1+C^{2}}}(|01\rangle-C|10\rangle)
$$

holds. Next, consider a product state $|u\rangle|w\rangle \in A_{\mathcal{Q}}$. We may represent the state $|u\rangle$ and $|w\rangle$ in the form

$$
\begin{aligned}
|u\rangle & =\frac{1}{\sqrt{1+|u|^{2}}}(|0\rangle+u|1\rangle) \\
|w\rangle & =\frac{1}{\sqrt{1+|w|^{2}}}(|0\rangle+w|1\rangle),
\end{aligned}
$$

for some complex constants $u, w \in \mathbb{C}$. As $\left\langle R_{\mathcal{Q}}|(|u\rangle \otimes|w\rangle)=0\right.$, we can then determine

$$
\begin{gathered}
w\langle 01 \mid 01\rangle-C u\langle 10 \mid 10\rangle=0 \\
\Longrightarrow \quad w=C u .
\end{gathered}
$$

Considering this, let $\rho_{\sigma} \otimes \tau_{\sigma}$ be some matching state of $\mathcal{Q}$. Suppose that Alice uses a mixed state for $\rho_{\sigma}$. Then, let $\rho_{\sigma}$ be some mixture of the states

$$
\begin{aligned}
& \left|\phi_{\sigma}\right\rangle=\frac{1}{\sqrt{1+\left|u_{\sigma}\right|^{2}}}\left(|0\rangle+u_{\sigma}|1\rangle\right) \\
& \left|\phi_{\sigma}^{\prime}\right\rangle=\frac{1}{\sqrt{1+\left|u_{\sigma}^{\prime}\right|^{2}}}\left(|0\rangle+u_{\sigma}^{\prime}|1\rangle\right),
\end{aligned}
$$

and let $\tau_{\sigma}$ be a (possibly trivial) mixture including the state

$$
\left|\psi_{\sigma}\right\rangle=\frac{1}{\sqrt{1+\left|w_{\sigma}\right|^{2}}}\left(|0\rangle+w_{\sigma}|1\rangle\right)
$$

Because $\left|\phi_{\sigma}\right\rangle\left|\psi_{\sigma}\right\rangle$ and $\left|\phi_{\sigma}^{\prime}\right\rangle\left|\psi_{\sigma}\right\rangle$ are both in $A_{\mathcal{Q}}$, it must be that $w_{\sigma}=C u_{\sigma}$ and $w_{\sigma}=C u_{\sigma}^{\prime}$. This is only possible if $u_{\sigma}=u_{\sigma}^{\prime}$, so that $\left|\phi_{\sigma}\right\rangle=\left|\phi_{\sigma}^{\prime}\right\rangle$. Then, $\rho_{\sigma}$ is a pure state. A similar analysis holds for Bob.

Next, we present the proof of Theorem 7 Implicit in the proof for the case where $u_{\alpha}, u_{\beta} \in \mathbb{C}$ is a derivation of the formula for $K_{\sigma}$ for canonical schemes that was shown previously, in Equation 18 
Proof of Theorem 9 - Without loss of generality, let $\mathcal{Q}$ be in canonical form. Let $\alpha \neq \beta$, and let

$$
\left|R_{\mathcal{Q}}\right\rangle=\frac{1}{\sqrt{1+C^{2}}}(|01\rangle-C|10\rangle),
$$

as before. We may express $\left|\phi_{\alpha}\right\rangle$ and $\left|\phi_{\beta}\right\rangle$ in terms of (extended) complex numbers, as in Equation 15 for instance, we have

$$
\left|\phi_{\alpha}\right\rangle=\frac{1}{\sqrt{1+\left|u_{\alpha}\right|^{2}}}\left(|0\rangle+u_{\alpha}|1\rangle\right),
$$

and similarly for $\left|\phi_{\beta}\right\rangle$. In the case where $\left|\phi_{\alpha}\right\rangle=|1\rangle$, we set $u_{\alpha}=\infty$ (using the one-point compactification of $\mathbb{C})$. We may then use Equation [16 to determine Bob's fingerprint states: for instance,

$$
\left|\psi_{\alpha}\right\rangle=\frac{1}{\sqrt{1+C^{2}\left|u_{\alpha}\right|^{2}}}\left(|0\rangle+C u_{\alpha}|1\rangle\right),
$$

and similarly for $\left|\psi_{\beta}\right\rangle$. Then, we will show that Equation 17 is satisfied for

$$
K_{\sigma}=\frac{C^{2}\left(1+\left|u_{\sigma}\right|^{2}\right)}{\left(1+C^{2}\left|u_{\sigma}\right|^{2}\right)\left(1+C^{2}\right)} .
$$

In the case $u_{\sigma}=\infty$, this expression has a well-defined limit of $K_{\sigma}=\frac{1}{1+C^{2}}$.

We have three cases: $u_{\alpha}=\infty$ (in which case $u_{\beta} \in \mathbb{C}$ ), $u_{\beta}=\infty$ (in which case $u_{\alpha} \in \mathbb{C}$ ), and $u_{\alpha}, u_{\beta} \in \mathbb{C}$. The first two cases are easy to prove; the last case of $u_{\alpha}, u_{b} \in$ $\mathbb{C}$ requires a little more analysis. Consider the value of $\left|\left\langle\phi_{\beta} \mid \phi_{\alpha}\right\rangle\right|^{2}:$

$$
\left|\left\langle\phi_{\beta} \mid \phi_{\alpha}\right\rangle\right|^{2}=\frac{\left|1+u_{\beta}{ }^{*} u_{\alpha}\right|^{2}}{\left(1+\left|u_{\alpha}\right|^{2}\right)\left(1+\left|u_{\beta}\right|^{2}\right)} .
$$

By subtracting both sides of the equation from 1, we obtain

$$
\begin{aligned}
1- & \left|\left\langle\phi_{\beta} \mid \phi_{\alpha}\right\rangle\right|^{2} \\
& =\frac{\left(1+u_{\alpha}{ }^{*} u_{\alpha}\right)\left(1+u_{\beta}{ }^{*} u_{\beta}\right)-\left(1+u_{\beta}{ }^{*} u_{\alpha}\right)\left(1+u_{\alpha}{ }^{*} u_{\beta}\right)}{\left(1+\left|u_{\alpha}\right|^{2}\right)\left(1+\left|u_{\beta}\right|^{2}\right)} \\
& =\frac{\left|u_{\alpha}-u_{\beta}\right|^{2}}{\left(1+\left|u_{\alpha}\right|^{2}\right)\left(1+\left|u_{\beta}\right|^{2}\right)}
\end{aligned}
$$

from which we conclude

$$
\frac{\left|u_{\alpha}-u_{\beta}\right|^{2}}{1+\left|u_{\alpha}\right|^{2}}=\left(1+\left|u_{\beta}\right|^{2}\right)\left(1-\left|\left\langle\phi_{\beta} \mid \phi_{\alpha}\right\rangle\right|^{2}\right) .
$$

We will make use of this equality in our analysis of $\left|\left\langle R_{\mathcal{Q}} \mid h_{\alpha \beta}\right\rangle\right|$. We may expand the expression for $\left|h_{\alpha \beta}\right\rangle$ in terms of the standard basis:

$$
\left|h_{\alpha \beta}\right\rangle=\frac{|00\rangle+C u_{\beta}|01\rangle+u_{\alpha}|10\rangle+C u_{\alpha} u_{\beta}|11\rangle}{\sqrt{1+\left|u_{\alpha}\right|^{2}} \sqrt{1+C^{2}\left|u_{\beta}\right|^{2}}} .
$$

Taking the inner product with $|R\rangle$, and squaring the absolute value, we obtain

$$
\left|\left\langle R_{\mathcal{Q}} \mid h_{\alpha \beta}\right\rangle\right|^{2}=\frac{C^{2}\left|u_{\alpha}-u_{\beta}\right|^{2}}{\left(1+\left|u_{\alpha}\right|^{2}\right)\left(1+C^{2}\left|u_{\beta}\right|^{2}\right)\left(1+C^{2}\right)} .
$$

Applying Equation 20 we can perform a substitution to obtain

$$
\begin{aligned}
\left|\left\langle R \mid h_{\alpha \beta}\right\rangle\right|^{2} & =\frac{C^{2}\left(1+\left|u_{\beta}\right|^{2}\right)}{\left(1+C^{2}\left|u_{\beta}\right|^{2}\right)\left(1+C^{2}\right)}\left(1-\left|\left\langle\phi_{\beta} \mid \phi_{\alpha}\right\rangle\right|^{2}\right) \\
& =K_{\beta}\left(1-\left|\left\langle\phi_{\beta} \mid \phi_{\alpha}\right\rangle\right|^{2}\right) .
\end{aligned}
$$

The theorem then holds.
[1] E. Kushilevitz, N. Nisan. Communication Complexity. Cambridge University Press (1997).

[2] A. C-C. Yao. Some complexity questions related to distributive computing. In Proceedings of 11th ACM STOC, pages 209-213 (1979).

[3] H. Buhrman, R. Cleve, J. Watrous, R. de Wolf. Quantum fingerprinting. In Physical Review Letters, Volume 87, Number 16, Article 167902 (2001). Also at quant-ph/0102001

[4] I. Newman, M. Szegedy. Public vs. private coin flips in one-round communication games. In Proceedings of the 28th Annual ACM Symposium on Theory of Computing, pages 561-570 (1996).

[5] H. Burhman, R. Cleve, A. Widgerson. Quantum vs. Clas- sical Communication and Computation. In Proceedings of the 30th Annual ACM Symposium on Theory of Computing, pages 63-68 (1998).

[6] R. Raz. Exponential separation of quantum and classical communication complexity. In Proceedings of the 31st Annual ACM Symposium on Theory of Computing, pages 358-367 (1999).

[7] S. Massar. Quantum Fingerprinting with a Single Particle. Los Alamos Pre-print Archive, quant-ph/0305112 (2003).

[8] A. Ambainis. Communication complexity in a 3-computer model. In Algorithmica, Volume 16, Number 3, pages 298301 (1996). 